\title{
Real-Time Energy-Efficient Actuation of Induction Motor Drives Using Approximate Dynamic Programming
}

\author{
Arne De Keyser ${ }^{\circledR}$, Hendrik Vansompel ${ }^{\circledR}$, and Guillaume Crevecoeur ${ }^{\circledR}$
}

\begin{abstract}
This article proposes a real-time control strategy for induction motor drives, harmonizing accurate torque, and flux tracking with energy efficiency. Common problems related to lengthy control horizons and the associated computational burden are dealt with by introducing a value function approximation in the model-predictive structure to quantify the impact of instantaneous decisions on future states. An augmented model of the drive is introduced to determine this value function approximation offline. The resulting optimization problem is implemented in a real-time environment and its effectiveness is established on an experimental setup for both steady state and dynamic load conditions. Experiments show that the proposed approach outperforms default model-based methods by up to $89.7 \%$, thus making it a promising real-time optimal control strategy in the domain of electric drives.
\end{abstract}

Index Terms-Approximate dynamic programming (ADP), electric drives, loss minimization, optimal control.

\section{INTRODUCTION}

$\mathbf{E}$ LECTRIC drives have become key in industrial applications as well as in electromobility and renewable energy systems. Dedicated control strategies are thus required, guaranteeing satisfactory actuation for the fast time scales related to the semiconductor power switches in the drive. Field-oriented control [1], [2] is one of the main standards in industry. Reliable operation is attained by controlling the stator current components in a synchronous reference frame. The associated control performance is however strongly correlated with the design of the intermediate proportional-integral controllers for the current components. A pronounced parameter dependency is furthermore present due to the inherent axis transformation.

Manuscript received August 28, 2020; revised October 21, 2020 and November 23, 2020; accepted December 3, 2020. Date of publication December 21, 2020; date of current version August 25, 2021. This work was supported in part by Flanders Make, the strategic research centre for the manufacturing industry, through the projects DIRAC and EMODO, and in part by the Special Research Fund under Grant 01N02716. (Corresponding author: Arne De Keyser.)

The authors are with the Department of Electromechanical, Systems, and Metal Engineering, Ghent University, 9052 Ghent, Belgium, and also with the EEDT-DC, Flanders Make, 3920 Lommel, Belgium (e-mail: arndkeys.dekeyser@ugent.be; hendrik.vansompel@ugent.be; guillaume.crevecoeur@ugent.be).

Color versions of one or more figures in this article are available at https://doi.org/10.1109/TIE.2020.3044791.

Digital Object Identifier 10.1109/TIE.2020.3044791
In contrast, no intermediate modulators or reference frame transformations are necessary when applying direct torque control (DTC) [3]. DTC furthermore exhibits superior transient properties and hence provides the capability of delivering a faster torque response in variable load scenarios. This may be a valuable asset for diverse industrial and manufacturing systems such as weaving looms. The associated torque ripple is nevertheless problematic, leading to acoustic noise and increased dissipation. Numerous possible improvements have therefore been proposed [4]-[10].

Nowadays, model-predictive control (MPC) has gathered a lot of research interest in the context of electric drives due to its capability to optimize arbitrary objective functions and integrate operational constraints [11], [12]. Reliable actuation may hence be combined with secondary objectives, e.g., optimization of the harmonic content [13] or loss minimization in the system [14], [15]. The latter is gaining importance in industry, as energyefficient operation is directly related to both economical and environmental aspects. Engaging MPC in the context of DTC results in socalled finite-set MPC [16]-[20]. The dynamic model of the drive is used to predict its future performance for a finite set of possible control inputs. For a traditional two-level voltage source inverter (2L-VSI), eight possible switching combinations have to be evaluated at each time step [16]-[18]. In case of supply by a three-level VSI (3L-VSI), this amounts to 27 options [13] [21]. At the fast time scales in power converters, a considerable prediction horizon is required to yield a reliable solution, hence leading to intractable computational demands. This necessitates the development of computationally efficient solvers that are approximately optimal for real-time control of electric drives.

Recent methods therefore focus on simplifying the associated long-term optimization problems [22]. In [23] the authors tackled the complex problem by reformulating it as an integer least-squares problem. The solution is then obtained by a branch-and-bound method called sphere decoding [24], [25]. Despite the severe reduction in computational requirements, no real-time solution for elevated time horizons has yet been provided. Approximate dynamic programming (ADP) [26] allows to reduce the computational requirements by summarizing the effect of control actions on future operation in a socalled value function. The number of necessary prediction steps, and thus computationally demanding model evaluations, is hereby restricted. State-of-the art methods may use iteratively updated 
value functions [27], [28]. Modifying the value function every time step nevertheless imposes an additional calculation load for real-time operation. A more computationally efficient tool is provided by [29], which allows the value function to be determined by offline solution of a semidefinite program (SDP). This framework has been successfully applied in simulation in the context of current control of electric drives [30].

To the authors' knowledge, ADP has however not been employed and experimentally validated for energy-efficient actuation of variable speed electric drives in contemporary industrial processes. The available knowledge concerning ADP cannot be directly applied to combine accurate reference tracking with energy-efficient operation. The governing state space formulation is nonlinear and the associated objective is generally a nonquadratic formulation with respect to the state variables. The value function can therefore not be defined offline using the methodology of [29]. Particular modifications to both the dynamic model of the drive and the ADP framework are required, after which a feasible value function approximation can be defined and employed.

This article presents a computationally efficient finite-set MPC strategy for energy-efficient actuation of induction motor (IM) drives. Real-time execution is rendered tractable by using ADP. The proposed methodology is applied to a drive consisting of a dc-source $V_{\mathrm{dc}}$, a two-level three-phase voltage source inverter $(2 \mathrm{~L}-\mathrm{VSI})$ and an IM rotating at a variable speed $\Omega$. In Section II, the dynamic model of the considered electric drive is introduced. Offline determination and online integration of the value function approximation are subsequently covered in more detail. Section III presents an experimental comparison of the introduced ADP-algorithm with respect to traditional DTC and competing finite-set MPC in terms of computational requirements and performance. The overall control performance is benchmarked for both steady-state operation and a realistic dynamic loading scenario. Section IV concludes this article.

\section{ADP FOR ENERGY-EFFICIENT ACTUATION}

Controlling the actuation is achieved by optimizing the gate signals in the inverter circuit. Providing reliable and energyefficient actuation for variable loads nevertheless requires dedicated modifications to existing ADP algorithms.

\section{A. Dynamic IM Drive Model}

To evaluate the impact of control decisions, a reliable dynamic model of the electric drive is required. The IM is modeled as a fourth-order state-space model in the stator currents $\boldsymbol{i}_{\mathrm{s}, \alpha \beta}$ and flux components $\psi_{\mathrm{s}, \alpha \beta}$ in the stationary $\alpha \beta$-frame. The state vector $\boldsymbol{x}$ is thus defined as $\boldsymbol{x}=\left[\begin{array}{llll}i_{\mathrm{s}, \alpha} & i_{\mathrm{s}, \beta} & \psi_{\mathrm{s}, \alpha} & \psi_{\mathrm{s}, \beta}\end{array}\right]^{T}$. The stator windings are connected to the legs of the inverter, whereas the rotor windings are short circuited. The dynamics of the machine are hence imposed by the inverter output voltage set $\boldsymbol{v}_{\alpha \beta}$.

$$
\dot{\boldsymbol{x}}(t)=\mathbf{A}(t) \boldsymbol{x}(t)+\mathbf{B} \boldsymbol{v}_{\alpha \beta}(t) .
$$

The characteristic system matrices $\mathbf{A}$ and $\mathbf{B}$ are

$$
\begin{aligned}
& \mathbf{A}(t)= \\
& {\left[\begin{array}{cccc}
\frac{L_{\mathrm{r}} R_{\mathrm{s}}+L_{\mathrm{s}} R_{\mathrm{r}}}{\kappa} & -N_{\mathrm{p}} \Omega(t) & -\frac{R_{\mathrm{r}}}{\kappa} & -\frac{L_{\mathrm{r}} N_{\mathrm{p}}}{\kappa} \Omega(t) \\
N_{\mathrm{p}} \Omega(t) & \frac{L_{\mathrm{r}} R_{\mathrm{s}}+L_{\mathrm{s}} R_{\mathrm{r}}}{\kappa} & \frac{L_{\mathrm{r}} N_{\mathrm{p}}}{\kappa} \Omega(t) & -\frac{R_{\mathrm{r}}}{\kappa} \\
-R_{\mathrm{s}} & 0 & 0 & 0 \\
0 & -R_{\mathrm{s}} & 0 & 0
\end{array}\right],} \\
& \mathbf{B}=\left[\begin{array}{cc}
-\frac{L_{\mathrm{r}}}{\kappa} & 0 \\
0 & -\frac{L_{\mathrm{r}}}{\kappa} \\
1 & 0 \\
0 & 1
\end{array}\right]
\end{aligned}
$$

with the notation $\kappa=L_{\mathrm{m}}^{2}-L_{\mathrm{r}} L_{\mathrm{s}}$. The symbols $R_{\mathrm{s}}$ and $R_{\mathrm{r}}$ refer to the stator and rotor resistances, respectively, whereas stator, mutual and rotor inductance are represented by $L_{\mathrm{s}}, L_{\mathrm{m}}$, and $L_{\mathrm{r}}$. $N_{\mathrm{p}}$ denotes the number of pole pairs. The possible variability of the rotational speed $\Omega$ is furthermore stressed by the explicit time notation, rendering matrix $\mathbf{A}$ time-variant.

The output torque of the drive $T$ is unambiguously determined by the state variables, according to

$$
T=\frac{3}{2} N_{\mathrm{p}}\left(i_{\mathrm{s}, \beta} \psi_{\mathrm{s}, \alpha}-i_{\mathrm{s}, \alpha} \psi_{\mathrm{s}, \beta}\right) .
$$

Based on the discrete nature of computer calculations, a discretized representation of (1) is nevertheless preferred. Due to the inherent time dependency of the system matrices, exact discretization of the model is not favorable. A first-order finite differencing approach is hence chosen. Sufficient accuracy is achieved at the small time steps encountered in electrical drives. A first-order discretized model with system matrices $\mathbf{A}_{k}^{\prime}$ and $\mathbf{B}^{\prime}$ and discrete time index $k$ is eventually obtained

$$
\boldsymbol{x}_{k+1}=\mathbf{A}_{k}^{\prime} \boldsymbol{x}_{k}+\mathbf{B}^{\prime} \boldsymbol{v}_{\alpha \beta, k} .
$$

\section{B. Approximate Dynamic Programming}

The dynamic drive equations provide reliable predictions of future system states. The cost of applying a certain switching sequence $\boldsymbol{S}_{\mathrm{abc}}$ can thus be predicted. This allows to rigorously compare the discrete set of possible switching states and elect the most promising sequence. Direct MPC (DMPC) finds the optimal switching state $\boldsymbol{S}_{\text {abc }}$ by minimizing an arbitrary loss function $\ell$ over a fixed horizon $N \in \mathbb{N}^{+}$. A discount factor $\gamma$ is hereby introduced. The initial state at the control instant is denoted by $\boldsymbol{x}_{0}$.

$$
\begin{array}{ll}
\underset{\boldsymbol{S}_{\mathrm{abc}, k}}{\operatorname{minimize}} & \sum_{k=1}^{N} \gamma^{k-1} \ell\left(\boldsymbol{x}_{k}\right) \\
\text { s.t. } & \boldsymbol{x}_{k+1}=\mathbf{A}_{k}^{\prime} \boldsymbol{x}_{k}+\mathbf{B}^{\prime} \boldsymbol{v}_{\alpha \beta, k} \\
& \left\|\boldsymbol{i}_{\mathrm{s}, \alpha \beta, k}\right\|_{\infty} \leq i_{\mathrm{lim}}, \boldsymbol{S}_{\mathrm{abc}, k} \in\{0,1\}^{3}
\end{array}
$$

The current in the $\alpha \beta$-frame is restricted to an arbitrary value $i_{\text {lim }}$, ensuring safe operation. In the remainder of this work, the maximum peak current is limited to the rated current. In the formulation of (5), obtaining close-to-optimal control actions would necessitate solving a minimization problem for lengthy 
time horizons. ADP overcomes this issue by summarizing the influence of future system behavior in a value function $V$, which depends on an arbitrary state vector $z$. The associated minimization problem is then reformulated.

$$
\begin{array}{ll}
\underset{\boldsymbol{S}_{\mathrm{abc}, k}}{\operatorname{minimize}} & \sum_{k=1}^{N} \gamma^{k-1} \ell\left(\boldsymbol{x}_{k}\right)+\gamma^{N} V\left(\boldsymbol{z}_{N}\right) \\
\text { s.t. } & \boldsymbol{x}_{k+1}=\mathbf{A}_{k}^{\prime} \boldsymbol{x}_{k}+\mathbf{B}^{\prime} \boldsymbol{v}_{\alpha \beta, k} \\
& \left\|\boldsymbol{i}_{\mathrm{s}, \alpha \beta, k}\right\|_{\infty} \leq i_{\mathrm{lim}}, \boldsymbol{S}_{\mathrm{abc}, k} \in\{0,1\}^{3}
\end{array}
$$

From a computational perspective, a fixed value function approximation $\hat{V}$ is favorable as this reduces the online calculation load. One can obtain an optimal value function approximation via the iterated Bellman inequality [29]

$$
\begin{array}{ll}
\operatorname{maximize} & \mathbf{E} \hat{V}_{0}(\boldsymbol{z}) \\
\text { s.t. } & \hat{V}_{i-1}\left(\boldsymbol{z}_{k}\right) \leq \ell_{j}(\boldsymbol{z})+\gamma \mathbf{E} \hat{V}_{i}\left(\boldsymbol{z}_{k+1}\right) \\
& \hat{V}_{0}=\hat{V}_{M}
\end{array}
$$

for every iteration $i \in \mathcal{I}=1 \ldots M$ and each discrete switching combination $j \in \mathcal{J}=1 \ldots 2^{3}$. The expected value-operator is herein characterized by $\mathbf{E}$. For the specific choice of quadratic candidate functions, $\hat{V}_{i}$ is cast into a computationally efficient format as a function of the coefficient matrices $\boldsymbol{F}_{i}$ and $\phi_{i}$ :

$$
\hat{V}_{i}(\boldsymbol{z})=\left[\begin{array}{c}
\boldsymbol{z} \\
1
\end{array}\right]^{T}\left[\begin{array}{cc}
\mathbf{F}_{i} & \mathbf{0} \\
\mathbf{0} & \phi_{i}
\end{array}\right]\left[\begin{array}{l}
\boldsymbol{z} \\
1
\end{array}\right] .
$$

Matrices $\mathbf{F}_{i}$ are hereby assumed to be symmetric.

\section{Loss Function Definition}

Favorable actuation combines accurate torque and flux tracking with energy efficiency. An appropriate stage cost $\ell$ is therefore symbolically introduced as a weighted sum of all intervening partial objectives

$$
\ell=\ell_{\mathrm{T}}+\delta_{\psi} \ell_{\psi}+\delta_{\mathrm{P}} \ell_{\mathrm{P}}
$$

with $\delta_{\psi}$ and $\delta_{\mathrm{P}}$ cost coefficients that may be adapted to reflect the importance of the different objectives. Torque and flux deviations are captured in $\ell_{\mathrm{T}}$ and $\ell_{\psi}$, respectively, whereas the power consumption intervenes in the cost term $\ell_{\mathrm{P}}$.

The cost of torque deviations $\ell_{T}$ is readily given by the quadratic difference between actual torque $T$ and the dynamic reference value $T^{*}$ at each time instant;

$$
\ell_{\mathrm{T}}\left(\boldsymbol{x}_{k}\right)=\left(T_{k}^{*}-T\left(\boldsymbol{x}_{k}\right)\right)^{2}
$$

and for a stator flux magnitude $\Psi_{\mathrm{s}}$ and a desired value $\Psi_{\mathrm{s}}^{*}$

$$
\ell_{\psi}\left(\boldsymbol{x}_{k}\right)=\left(\Psi_{\mathrm{s}, k}^{2}-\Psi^{*^{2}}\right)^{2}
$$

The energy-related $\operatorname{cost} \ell_{\mathrm{P}}$ is proposed in a straightforward manner when considering the total power consumption $P_{\mathrm{i}}$ :

$$
\ell_{\mathrm{P}}\left(\boldsymbol{x}_{k}, \boldsymbol{S}_{\mathrm{abc}, k}\right)=\boldsymbol{v}_{\mathrm{abc}, j, k}^{T} \boldsymbol{i}_{\mathrm{abc}}\left(\boldsymbol{x}_{k}\right) \triangleq 2 \boldsymbol{q}_{j}^{P^{T}} \boldsymbol{x}_{k} .
$$

The three-phase currents $\boldsymbol{i}_{\text {abc }}$ are directly related to the state variables, whereas the inverter state unambiguously defines the voltage output $\boldsymbol{v}_{\mathrm{abc}, j}$. This dependency on the state and the discrete mode $j$ is incorporated in $\boldsymbol{q}_{j}^{P}$. Inverter losses are neglected but can be included in future work [18]. Iron losses are furthermore not considered. While both loss mechanisms are not modeled, they are inherently present in experiments.

One can intuitively deduce that the objectives, being torque and flux tracking, i.e., (10) and (11), and energy efficiency (12), are counteracting. From an energetic perspective, minimizing the consumed power corresponds to an absence of current and consequently a situation of no machine excitation. This obviously impedes an accurate reference tracking in terms of torque and flux. Higher values for $\delta_{\mathrm{P}}$ in (9) are linked to an energy-efficient performance, while lower values favor more accurate actuation. The flux-related coefficient $\delta_{\psi}$ balances deviations in both torque and flux and can theoretically be set to zero. The algorithm is then able to overrule the imposed flux reference without any punishment. For a shortterm prediction, this however leads to excessive currents in the windings.

\section{Augmented IM Drive Model}

In order to cast the optimization problem into the ADPframework and render (7) tractable for quadratic candidate functions (8), the stage cost (9) needs to be translated into its equivalent quadratic form. For the generalized state vector $\boldsymbol{z}$, an appropriate stage cost $\ell$ is thus of the following form:

$$
\ell_{j}(\boldsymbol{z})=\left[\begin{array}{c}
\boldsymbol{z} \\
1
\end{array}\right]^{T}\left[\begin{array}{cc}
\mathbf{Q} & \boldsymbol{q}_{j} \\
\boldsymbol{q}_{j}^{T} & l_{j}
\end{array}\right]\left[\begin{array}{l}
\boldsymbol{z} \\
1
\end{array}\right]
$$

with $j \in \mathcal{J}$ referring to the possible switching combinations of the inverter. For each switching mode, the voltage vector $\boldsymbol{v}_{a b c, j}$ is thus uniquely defined. Matrix $\mathbf{Q}$ is assumed to be symmetrical and positive semidefinite. No specific restrictions on the other matrices are imposed [29]. Both $\ell_{\psi}$ and $\ell_{\mathrm{T}_{\mathrm{IM}}}$ however behave as fourth-order functions in the state variables $\boldsymbol{x}$, hence requiring a redefinition of the state vector.

The introduction of a supplementary state vector $\boldsymbol{x}^{+}$provides the means to formulate the dynamic torque $T$ and flux magnitude $\Psi_{\mathrm{S}}$ for a given set of input voltages $\boldsymbol{v}_{\mathrm{S}, \alpha \beta, j}$ and a constant speed $\Omega$, inspired by [4].

$$
\boldsymbol{x}^{+}=\left[\begin{array}{c}
i_{\mathrm{s}, \beta} \psi_{\mathrm{s}, \alpha}-i_{\mathrm{s}, \alpha} \psi_{\mathrm{s}, \beta} \\
i_{\mathrm{s}, \alpha} \psi_{\mathrm{s}, \alpha}+i_{\mathrm{s}, \beta} \psi_{\mathrm{s}, \beta} \\
\psi_{\mathrm{s}, \alpha}^{2}+\psi_{\mathrm{s}, \beta}^{2} \\
i_{\mathrm{s}, \alpha}^{2}+i_{\mathrm{s}, \beta}^{2}
\end{array}\right]
$$

After the necessary mathematical manipulations, the dynamic behavior is determined by considering the augmented state vector $\boldsymbol{z}=\left[\begin{array}{llll}\boldsymbol{x}^{+} & \boldsymbol{x} & T^{*} & \Psi_{\mathrm{s}}^{*}\end{array}\right]^{T}$.

$$
\frac{\mathrm{d} \boldsymbol{x}^{+}}{\mathrm{d} t}=\mathbf{A}_{j}^{+} \boldsymbol{z} .
$$

The additional state matrix $\mathbf{A}_{j}^{+} \in \mathbb{R}^{4 \times 10}$ is hereby defined as a function of the voltage components $v_{\alpha, j}$ and $v_{\beta, j}$ in the discrete 
switching mode $j$ and is given by (16) shown at the bottom of this page.

The torque and flux references are hence included in the augmented state vector to simplify the convex cost definition. For the small time constants encountered in electric drives, these quantities are assumed constant. The speed $\Omega$ is reliably supposed to be fixed as well. The augmented drive model is eventually represented by a tenth-order state space system

$$
\frac{\mathrm{d} \boldsymbol{z}}{\mathrm{d} t}=\left[\begin{array}{cccc}
\mathbf{0} & \mathbf{A}_{j}^{+} & \\
\mathbf{0} & \mathbf{A} & \mathbf{0} & \mathbf{0} \\
\mathbf{0} & \mathbf{0} & 0 & 0 \\
\mathbf{0} & \mathbf{0} & 0 & 0
\end{array}\right] \boldsymbol{z}+\left[\begin{array}{c}
\mathbf{0} \\
\mathbf{B} \\
0 \\
0
\end{array}\right] \boldsymbol{v}_{\alpha \beta, j}
$$

or equivalently, implicitly defining $\tilde{\mathbf{A}}_{j}$ and $\tilde{\boldsymbol{b}}_{j}$,

$$
\frac{\mathrm{d} \boldsymbol{z}}{\mathrm{d} t} \triangleq \tilde{\mathbf{A}}_{j} \boldsymbol{z}+\tilde{\boldsymbol{b}}_{j}
$$

The respective cost terms (10) and (11) are then readily rewritten as a quadratic form in the augmented state $z$ :

$$
\ell_{\mathrm{T}}\left(\boldsymbol{z}_{k}\right) \triangleq \boldsymbol{z}_{k}^{T} \mathbf{Q}^{T} \boldsymbol{z}_{k} ; \ell_{\psi}\left(\boldsymbol{z}_{k}\right) \triangleq \boldsymbol{z}_{k}^{T} \mathbf{Q}^{\psi} \boldsymbol{z}_{k} .
$$

Matrices $\mathbf{Q}^{\psi}$ and $\mathbf{Q}^{T}$ are hereby positive semidefinite, as they correspond to quadratic deviations. The total state-cost matrix $\mathbf{Q}$ in (13) then adds up to

$$
\mathbf{Q}=\mathbf{Q}^{T}+\delta_{\psi} \mathbf{Q}^{\psi}
$$

and, recalling (12),

$$
\boldsymbol{q}_{j}=\delta_{\mathrm{P}} \boldsymbol{q}_{j}^{P}
$$

\section{E. Real-Time Energy-Efficient Actuation}

Based on a discretization of the augmented state evolution given in (18), with corresponding matrices $\tilde{\mathbf{A}}_{j}^{\prime}$ and $\tilde{\boldsymbol{b}}_{j}^{\prime}$, and the definition of the quadratic value function approximations (8), one can explicitly calculate the expected value of the future state for the set of Bellman inequalities in (7).

$$
\mathbf{E} \hat{V}_{i}\left(\boldsymbol{z}_{k+1}\right)=\mathbf{E} \hat{V}_{i}\left(\tilde{\mathbf{A}}_{j}^{\prime} \boldsymbol{z}_{k}+\tilde{\boldsymbol{b}}_{j}^{\prime}\right)
$$

After the necessary calculations, one eventually attains [29]

$$
\mathbf{E} \hat{V}_{i}\left(\boldsymbol{z}_{k+1}\right)=\left[\begin{array}{c}
\boldsymbol{z}_{k} \\
1
\end{array}\right]^{T}\left[\begin{array}{cc}
\mathbf{H}_{i, j} & \boldsymbol{h}_{i, j} \\
\boldsymbol{h}_{i, j}^{T} & \eta_{i, j}
\end{array}\right]\left[\begin{array}{c}
\boldsymbol{z}_{k} \\
1
\end{array}\right]
$$

with

$$
\mathbf{H}_{i, j}=\tilde{\mathbf{A}}_{j}^{\prime T} \mathbf{F}_{i} \tilde{\mathbf{A}}_{j}^{\prime} ; \boldsymbol{h}_{i, j}=\tilde{\mathbf{A}}_{j}^{\prime T} \mathbf{F}_{i} \tilde{\boldsymbol{b}}_{j}^{\prime} ; \eta_{i, j}=\tilde{\boldsymbol{b}}_{j}^{\prime T} \mathbf{F}_{i} \tilde{\boldsymbol{b}}_{j}^{\prime}+\phi_{i} .
$$

By inserting (8), (13), and (23) in the inequality constraints of problem (7), following linear matrix inequality (LMI) in the

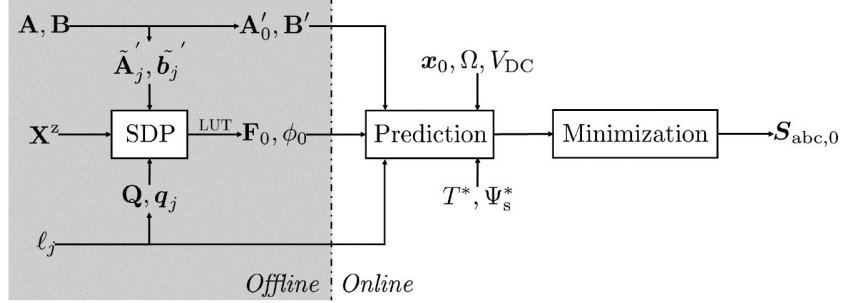

Fig. 1. Overview of the ADP-algorithm. The value function approximation is defined in an offline phase for various speeds. A brute force minimization of the switching sequence is then executed online for given reference values in torque and flux.

unknown coefficient matrices is obtained

$$
\left[\begin{array}{cc}
\mathbf{Q}+\gamma \mathbf{H}_{i, j}-\mathbf{F}_{i-1} & \boldsymbol{q}_{j}+\gamma \boldsymbol{h}_{i, j} \\
\boldsymbol{q}_{j}^{T}+\gamma \boldsymbol{h}_{i, j}^{T} & \gamma \eta_{i, j}-\phi_{i-1}
\end{array}\right] \geq 0 .
$$

An analogous LMI is composed for all modes $j$. Additional constraints are imposed by restricting $\boldsymbol{S}_{\mathrm{abc}} \in\{0,1\}^{3}$, which is inherently fulfilled by explicitly enumerating the distinct possible operating modes. The limitation on the current components can be integrated by using the $\mathcal{S}$-procedure [29].

As a direct consequence of the quadratic candidate functions for $\hat{V}_{i}$, the general formulation of (7) can be replaced by a computationally efficient SDP in the corresponding coefficient matrices $\mathbf{F}_{0}$ and $\phi_{0}$.

$$
\begin{array}{ll}
\underset{\mathbf{F}_{0}, \phi_{0}}{\operatorname{maximize}} & \mathbf{E} \hat{V}_{0}(\tilde{\boldsymbol{x}})=\operatorname{Tr}\left(\mathbf{F}_{0} \mathbf{X}^{z}\right)+\phi_{0} \\
\text { s.t. } & (25), j \in \mathcal{J} ; i \in \mathcal{I} \\
& \hat{V}_{0}=\hat{V}_{M} .
\end{array}
$$

The Tr-function denotes the trace of a square matrix. The state covariance $\mathbf{X}^{z}$ should furthermore be representative for the distribution of the state space during operation. A smaller covariance leads to an accurate approximation close to the mean values, whereas larger values tend to describe an extensive area in less detail. A well-founded choice is therefore decisive for the performance of the algorithm.

The overall control problem hence encompasses two separate stages as depicted in Fig. 1. In the first stage, the optimal quadratic value function approximation $\hat{V}_{0}$ is determined offline in terms of $\mathbf{F}_{0}$ and $\phi_{0}$ based on (26). As previous considerations are valid at a single value of the rotational speed, due to the dependency on $\tilde{\mathbf{A}}_{j}^{\prime}$, the SDP is solved at a range of discrete speeds $\mathcal{W}$ in the operation area. The resulting coefficient matrices $\mathbf{F}_{0}$ and $\phi_{0}$ for the value function approximations are then stored in look-up tables (LUT). Algorithm 1 describes this offline procedure in more detail.

During online operation of the drive, all discrete options are explicitly enumerated, which is feasible for limited prediction

$$
\mathbf{A}_{j}^{+}=\left[\begin{array}{cccccccccc}
\frac{L_{\mathrm{r}} R_{\mathrm{s}}+L_{\mathrm{s}} R_{\mathrm{r}}}{\kappa} & N_{\mathrm{p}} \Omega & \frac{L_{\mathrm{r}} N_{\mathrm{p}}}{\kappa} \Omega & 0 & -v_{\beta, j} & v_{\alpha, j} & -\frac{L_{\mathrm{r}}}{\kappa} v_{\beta, j} & \frac{L_{\mathrm{r}}}{\kappa} v_{\alpha, j} & 0 & 0 \\
-N_{\mathrm{p}} \Omega & \frac{L_{\mathrm{r}} R_{\mathrm{s}}+L_{\mathrm{s}} R_{\mathrm{r}}}{\kappa} & -\frac{R_{\mathrm{r}}}{\kappa} & -R_{\mathrm{s}} & v_{\alpha, j} & v_{\beta, j} & -\frac{L_{\mathrm{r}}}{\kappa} v_{\alpha, j} & -\frac{L_{\mathrm{r}}}{\kappa} v_{\beta, j} & 0 & 0 \\
0 & -2 R_{\mathrm{s}} & 0 & 0 & 0 & 0 & 2 v_{\alpha, j} & 2 v_{\beta, j} & 0 & 0 \\
\frac{2 L_{\mathrm{r}} N_{\mathrm{p}}}{\kappa} \Omega & -\frac{2 R_{\mathrm{r}}}{\kappa} & 0 & \frac{2\left(L_{\mathrm{r}} R_{\mathrm{s}}+L_{\mathrm{s}} R_{\mathrm{r}}\right)}{\kappa} & -\frac{2 L_{\mathrm{r}}}{\kappa} v_{\alpha, j} & -\frac{2 L_{\mathrm{r}}}{\kappa} v_{\beta, j} & 0 & 0 & 0 & 0
\end{array}\right]
$$




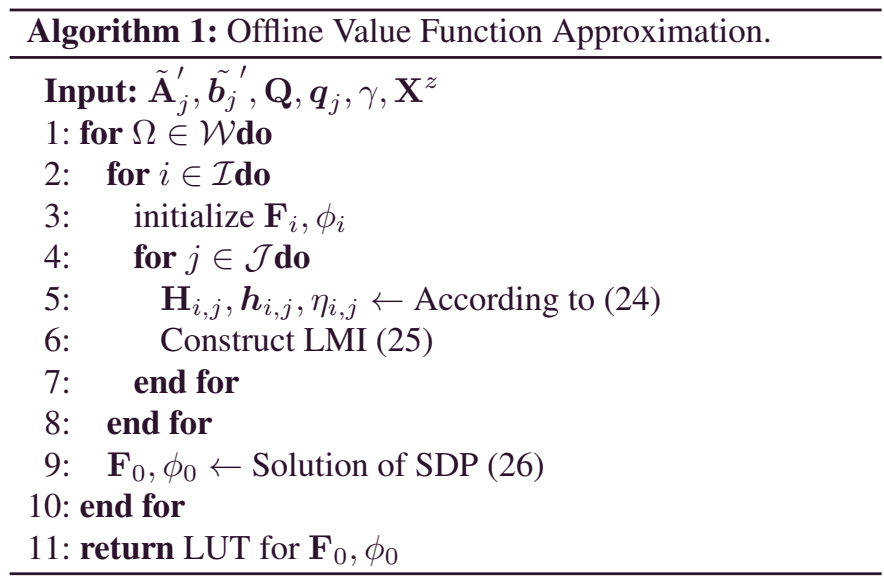

horizons $N$. Long-term effects are taken into account by evaluating the value function approximation $\hat{V}_{\Omega}$ at the final state $\boldsymbol{z}_{N}$. The value function approximation $\hat{V}_{\Omega}$ for an instantaneous speed $\Omega$ is computed using linear interpolation in the previously stored LUT. A coarser grid of rotational speeds hence leads to less accurate interpolation results but reduces the required memory usage. The online minimization problem is therefore formulated as

$$
\begin{array}{ll}
\underset{\boldsymbol{S}_{\mathrm{abc}, k}}{\operatorname{minimize}} & \sum_{k=1}^{N} \gamma^{k-1} \ell_{j}\left(\boldsymbol{x}_{k}\right)+\gamma^{N} \hat{V}_{\Omega}\left(\boldsymbol{z}_{N}\right) \\
\text { s.t. } & \boldsymbol{x}_{k+1}=\mathbf{A}_{k}^{\prime} \boldsymbol{x}_{k}+\mathbf{B}^{\prime} \boldsymbol{v}_{\alpha \beta, k} \\
& \left\|\boldsymbol{i}_{\mathrm{s}, \alpha \beta, k}\right\|_{\infty} \leq i_{\mathrm{lim}}, \boldsymbol{S}_{\mathrm{abc}, k} \in\{0,1\}^{3} .
\end{array}
$$

The augmented state-space vector $z$ is only necessary for the evaluation of $\hat{V}_{\Omega}$. Its dynamical evolution is not required during the online phase. The specific implementation of the online minimization problem for single-step predictions $(N=1)$ is provided in Algorithm 2. The arbitrary cost $\ell_{s c}$ is hereby introduced to translate the constraint on the maximum admissible current magnitude $i_{\lim }$ into its equivalent soft constraint. The num2bin-operator converts the discrete operating mode $j$ into the appropriate binary vector.

Extensions of the algorithm toward multistep predictions may be carried out in a straightforward manner. The number of discrete options to be evaluated then increases exponentially by a factor eight.

\section{EXPERIMENTAL VALIDATION}

A profound experimental verification of the introduced algorithm is indispensable as to demonstrate real-time feasibility and rigorously assess the achievable progress for industrial applications. Secondary loss phenomena, e.g., inverter losses and iron losses, are furthermore not included in the models of the drive but are inherently present in experimental data.

\section{A. Experimental Setup}

The introduced implementation is experimentally validated on a $5.5 \mathrm{~kW}$ asynchronous motor in the multipurpose laboratory setup depicted in Fig. 2. In this setup two $5.5 \mathrm{~kW}, 3000 \mathrm{rpm}$ IE3
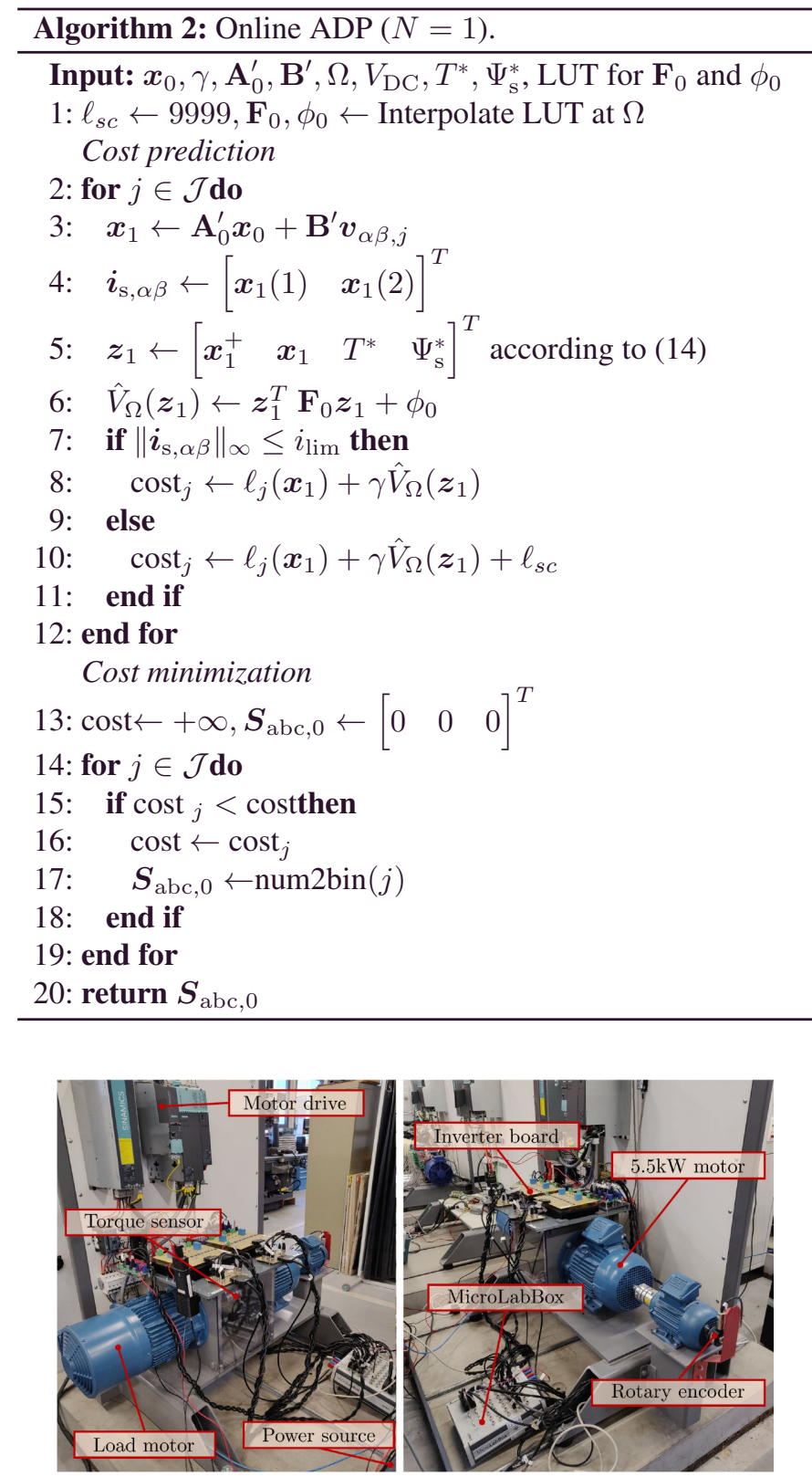

Fig. 2. Overview of the multipurpose experimental setup with annotation of the main components and sensing equipment.

asynchronous motors are placed in a back-to-back configuration. A Lorenz DR-2112 torque sensor is capable of measuring the torque transferred between both motors. The third smaller motor on the right side is not used in this experiment but is equipped with a rotary encoder.

The power stage of the inverter includes an Infineon FS50R12KT4 B15 three-phase full-bridge IGBT module, current measurements and a $2200 \mu \mathrm{F}$ dc-link capacitor. Power is supplied by a Delta Elektronika SM 500-CP-90 bidirectional power source. The drawn current at the inverter side and the dcvoltage can be monitored. A dSPACE rapid control prototyping platform MicroLabBox with integrated real-time processor and a Xilinx Kintex-7 XC7K325T field-programmable gate array (FPGA) is used to implement to low-level motor control strategy [31]. The load motor is connected to a Siemens Sinamics S120 
TABLE I

IDENTIFIED PARAMETERS OF THE $5.5 \mathrm{~kW}-3000$ RPM MOTOR

\begin{tabular}{c|c|c|c|c|c}
\hline $\boldsymbol{T}_{\mathbf{r}}[\mathrm{Nm}]$ & $\boldsymbol{R}_{\mathbf{S}}[\Omega]$ & $\boldsymbol{R}_{\mathbf{r}}[\Omega]$ & $\boldsymbol{L}_{\mathbf{s}}[\mathrm{H}]$ & $\boldsymbol{L}_{\mathbf{r}}[\mathrm{H}]$ & $\boldsymbol{L}_{\mathbf{m}}[\mathrm{H}]$ \\
\hline \hline 17.5 & 0.8917 & 0.3586 & 0.1570 & 0.1569 & 0.1525 \\
\hline
\end{tabular}

TABLE II

REFERENCE VALUES FOR THE PU-VARIABLES

\begin{tabular}{c|c|c|c|c}
\hline$V_{\mathrm{b}}$ & $\omega_{\mathrm{b}}$ & $T_{\mathrm{b}}$ & $I_{\mathrm{b}}$ & $Z_{\mathrm{b}}$ \\
\hline \hline$V_{\mathrm{DC}} / 2$ & $2 \pi f_{\mathrm{r}}$ & $N_{\mathrm{p}} P_{\mathrm{r}} / \omega_{\mathrm{b}}$ & $T_{\mathrm{b}} \omega_{\mathrm{b}} / V_{\mathrm{b}}$ & $V_{\mathrm{b}} / I_{\mathrm{b}}$ \\
\hline
\end{tabular}

motor module. The stator flux is estimated using a discrete constant gain observer [32] with a negative gain $b$ of $5.0 \cdot 10^{-5}$.

The parameters of the drive, with a power rating $P_{\mathrm{r}}$ equaling $5.5 \mathrm{~kW}$ and a single pole pair, are summarized in Table I. The rated frequency $f_{r}$ is $50 \mathrm{~Hz}$, the control frequency equals $10 \mathrm{kHz}$ $(\Delta t=100 \mu \mathrm{s})$ and the dc-link voltage $V_{\mathrm{dc}}$ is fixed at $400 \mathrm{~V}$. The rated torque is denoted by $T_{\mathrm{r}}$.

From an implementation perspective, it is nevertheless more favorable to translate these values into equivalent pu-quantities. Reference quantities for voltage, torque, current and impedance are respectively denoted by $V_{b}, T_{b}, I_{b}$, and $Z_{b}$, whereas the inverse time base is symbolized as $\omega_{b}$. Table II provides an overview of the common base references.

\section{B. Hardware Implementation}

The intended actuation of the experimental drive aims at tracking variable torque profiles with minimal energy consumption, as reflected in the cost definition of (9). Whereas the torque reference is externally imposed by the load, the associated flux setpoint remains a degree of freedom. To facilitate further energy-efficient actuation, a static variable flux level is chosen to minimize steady-state power losses [2]. The optimal stator flux reference $\Psi_{\mathrm{s}}^{*}$ is hence directly correlated to the actual torque demand $T^{*}$ according to

$$
\Psi_{\mathrm{s}}^{*}=\sqrt{\frac{L_{\mathrm{s}}^{2}}{L_{\mathrm{m}}^{2}} \Psi_{\mathrm{r}}^{* 2}+\frac{4}{9} \frac{1}{N_{\mathrm{p}}^{2}} \frac{\left(L_{\mathrm{m}}^{2}-L_{\mathrm{s}} L_{\mathrm{r}}\right)^{2}}{L_{\mathrm{m}}^{2}} \frac{T^{*^{2}}}{\Psi_{\mathrm{r}}^{*}}}
$$

with the optimal rotor flux magnitude $\Psi_{\mathrm{r}}^{*}$ equaling [2]

$$
\Psi_{\mathrm{r}}^{*}=\sqrt{\frac{2}{3} \frac{L_{\mathrm{r}}}{N_{\mathrm{p}}}\left|T^{*}\right| \sqrt{\left(1+\frac{R_{\mathrm{r}}}{R_{\mathrm{s}}} \frac{L_{\mathrm{m}}^{2}}{L_{\mathrm{r}}^{2}}\right)} .}
$$

Note that a more dynamic flux trajectory might entail better results. This would however require additional computational resources and is hence kept for future research. In practical applications, an upper and lower limit is imposed on the desired flux level. For low fluxes, controllability of the drive might become troublesome. If the rotor flux becomes too high, magnetic saturation occurs. The flux reference is hence maintained between 0.4 and $0.9 \mathrm{~Wb}$.

Based on (28), the cost coefficient for flux deviations $\delta_{\psi}$ is defined in (30) to balance flux and torque errors. The definition of $\delta_{\mathrm{P}}$ is somewhat more arbitrary and is empirically set to 0.025 in pu. Higher values are shown to impede physical actuation as
TABLE III

EXPECTED LATENCY AND ESTIMATED RESOURCE USAGE FOR THE REAL-TIME IMPLEMENTATION OF THE DIFFERENT CONTROL STRATEGIES

\begin{tabular}{c||c|c|c|c|c}
\hline & $\boldsymbol{t}_{\mathrm{ex}}[\mu \mathrm{s}]$ & BRAM & DSP & FF & LUT \\
\hline \hline DTC & 0.22 & 0 & 35 & 774 & 486 \\
MPC $^{1}$ & 1.68 & 0 & 80 & 6134 & 6672 \\
MPC $^{\mathbf{2}}$ & 8.09 & 0 & 162 & 9535 & 15772 \\
MPC $^{\mathbf{3}}$ & 28.38 & 0 & 84 & 14432 & 14501 \\
\hline ADP $^{1}$ & 1.90 & 0 & 239 & 9754 & 9083 \\
\hline
\end{tabular}

energy savings are overvalued on the short term.

$$
\delta_{\mathrm{P}}=0.025 ; \delta_{\psi}=T^{*^{2}} / \Psi_{\mathrm{s}}^{*^{4}}=0.0315 .
$$

In the offline stage, the value function approximations are then defined based on (26). A representative state covariance $\mathbf{X}^{z}$ is composed based on prior data of default DMPC-approaches. The discount factor $\gamma$ is fixed at 0.95 . Determining the value function approximation is executed for 30 iterations $(M=30)$ and for a uniform speed from standstill to $1700 \mathrm{rpm}$ with a step of $100 \mathrm{rpm}$. The offline SDP is implemented in MATLAB using YALMIP [33] and solved with MOSEK [34]. The LUT for the value function approximation is hence constructed for a total of 18 speed values.

At each control instant, the real-time optimization problem (27) is solved for a predefined prediction horizon $N$. A combination of Vivado HLS 2016.4 and Xilinx System Generator 2016.4 is employed to synthesize the algorithm on the FPGA. Three state-of-the-art DMPC-approaches are considered with prediction horizons ranging from one to three, i.e., $\mathrm{MPC}^{1}$, $\mathrm{MPC}^{2}$, and $\mathrm{MPC}^{3}$. The associated performances are compared to an $\mathrm{ADP}$-algorithm with single-step prediction $\left(\mathrm{ADP}^{1}\right)$ and traditional DTC [3], with an optimized stator flux reference.

The eventual resource utilization of the different algorithms is summarized in Table III in terms of block RAM (BRAM), DSP, flip-flops (FF) and LUT. A fixed point representation with a total of $25 \mathrm{~b}$, of which $20 \mathrm{~b}$ reflect the decimal part, is used. Further reduction of the number of bits is not possible without loss of accuracy. The latency $t_{\mathrm{ex}}$ of $\mathrm{ADP}^{1}$ implementation is comparable to the single-step $\mathrm{MPC}^{1}$, whereas the $\mathrm{ADP}^{1}$ algorithm is expected to significantly outperform MPC ${ }^{1}$. This obviously comes with a more pronounced resource usage. The required resources are however amply available.

\section{Experimental Results}

Experimental data are gathered for both steady-state operation and a dynamic load, representative for industrial scenarios.

1) Steady-State Operation: The $5.5 \mathrm{~kW}$-motor is initially not powered, resulting in an initial state that approximately coincides with the zero state. The load motor is driving the shaft at a constant speed. The torque reference is then changed to $0.5 T_{\mathrm{r}}$. After a delay of $10 \mathrm{~s}, 60 \mathrm{~s}$ of experimental steady-state data is gathered. Such experiments are carried out at two distinct speeds, i.e., 300 and $1500 \mathrm{rpm}$.

The response of the main electrical quantities is summarized in Fig. 3 for the drive rotating at $300 \mathrm{rpm}$. Traditional DTC demonstrates a pronounced torque ripple at the chosen sampling frequency of $25 \mathrm{kHz}$. For MPC ${ }^{1}$, one observes a situation of 

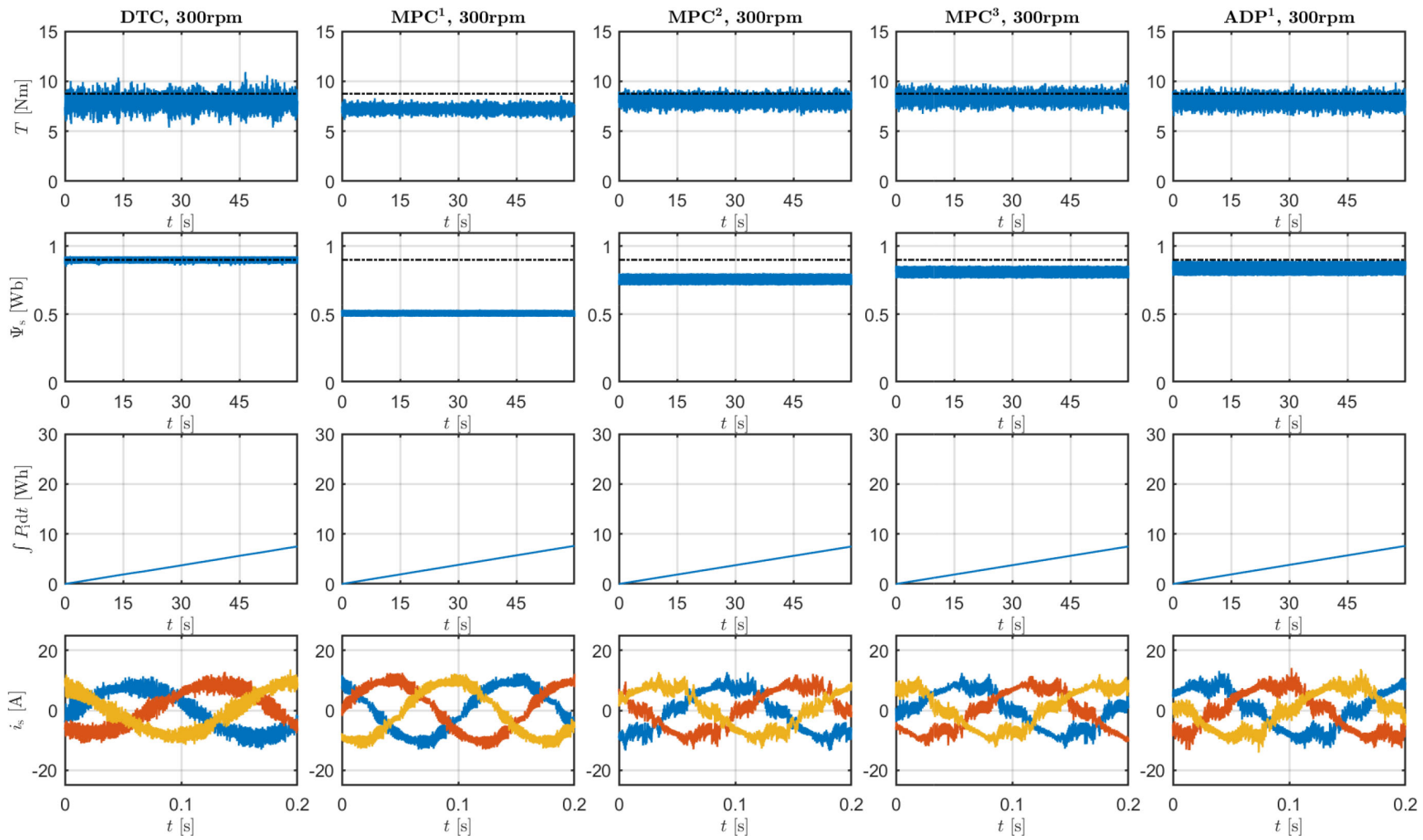

Fig. 3. Experimental steady-state response of representative quantities, i.e., torque, stator flux, consumed energy, and stator current components, for the considered $5.5 \mathrm{~kW}$-motor rotating at $300 \mathrm{rpm}$ and actuated using the five alternative controllers.

persistent underactuation. The consumed energy is reduced by delivering less torque. If only a limited prediction window is engaged, the negative impact on the long-term in terms of total cost for torque deviations is not taken into account. A closer match with the reference torque is attained by either increasing the prediction horizon or incorporating the value function approximation. A similar remark can be made with respect to the attained flux level. The default model-based controllers attempt to reduce the total energy consumption by limiting the flux build-up, which is beneficial on the short term. The resulting steady-state flux level is however consistently below the reference value, inevitably leading to a higher flux-related cost over the course of the complete experiment. This undesired behavior is eliminated by incorporating the value function approximation. Differences with respect to energy consumption are hard to distinguish. At lower speeds, the waveforms of the stator current components $\boldsymbol{i}_{\mathrm{s}, \mathrm{abc}}$ are relatively sinusoidal. More accurate actuation however comes at the cost of a slightly higher harmonic content, with an average total harmonic distortion monotonously increasing from $4.7 \%$ for $\mathrm{MPC}^{1}$ to $11.1 \%$ for $\mathrm{ADP}^{1}$. The average number of switching transitions per second furthermore equals $2.7 \mathrm{kHz}$ for $\mathrm{ADP}^{1}$, compared to $1.7 \mathrm{kHz}$ for $\mathrm{MPC}^{1}$. Switching losses are hence more pronounced. These losses are however most prominent in the case of traditional DTC, introducing an average switching frequency of $3.9 \mathrm{kHz}$.

Similar observations can be made for the asynchronous drive rotating at $1500 \mathrm{rpm}$, for which the experimental data are gathered in Fig. 4. The total energy consumption is considerably increased, as the mechanical power demand increases by a factor five.
TABLE IV

Detailed OVerview of the Averaged Cost for Ten Independent EXPERIMENTS ON THE $5.5 \mathrm{~kW}$-MOTOR AT 300 RPM

\begin{tabular}{c||c|c|c|c|c}
\hline & DTC & MPC $^{\mathbf{1}}$ & MPC $^{2}$ & MPC $^{3}$ & ADP $^{1}$ \\
\hline \hline$\Sigma \boldsymbol{\ell}_{\mathbf{T}}$ & $1.05 \cdot 10^{6}$ & $1.64 \cdot 10^{6}$ & $4.64 \cdot 10^{5}$ & $2.79 \cdot 10^{5}$ & $5.06 \cdot 10^{5}$ \\
$\Sigma \boldsymbol{\ell}_{\boldsymbol{\psi}}$ & $0.85 \cdot 10^{3}$ & $1.83 \cdot 10^{5}$ & $3.44 \cdot 10^{4}$ & $1.39 \cdot 10^{4}$ & $7.43 \cdot 10^{3}$ \\
$\Sigma \boldsymbol{\ell}_{\mathbf{P}}$ & $2.78 \cdot 10^{8}$ & $2.71 \cdot 10^{8}$ & $2.67 \cdot 10^{8}$ & $2.68 \cdot 10^{8}$ & $2.71 \cdot 10^{8}$ \\
\hline$\Sigma \boldsymbol{\ell}$ & $1.49 \cdot 10^{6}$ & $1.27 \cdot 10^{7}$ & $2.86 \cdot 10^{6}$ & $1.47 \cdot 10^{6}$ & $1.32 \cdot 10^{6}$ \\
\hline
\end{tabular}

TABLE V

Detailed OVerview of the AVERAged Cost for TEN INDEPENDENT EXPERIMENTS ON THE $5.5 \mathrm{~kW}$-MOTOR AT $1500 \mathrm{RPM}$

\begin{tabular}{c||c|c|c|c|c}
\hline & DTC & MPC $^{1}$ & MPC $^{2}$ & MPC $^{3}$ & ADP $^{1}$ \\
\hline \hline$\Sigma \boldsymbol{\ell}_{\mathbf{T}}$ & $1.94 \cdot 10^{6}$ & $4.42 \cdot 10^{6}$ & $2.50 \cdot 10^{5}$ & $2.58 \cdot 10^{5}$ & $2.87 \cdot 10^{5}$ \\
$\Sigma \boldsymbol{\ell}_{\boldsymbol{\psi}}$ & $0.33 \cdot 10^{3}$ & $3.78 \cdot 10^{4}$ & $2.72 \cdot 10^{3}$ & $1.41 \cdot 10^{3}$ & $2.28 \cdot 10^{3}$ \\
$\Sigma \boldsymbol{\ell}_{\mathbf{P}}$ & $8.01 \cdot 10^{8}$ & $9.29 \cdot 10^{8}$ & $1.04 \cdot 10^{9}$ & $1.04 \cdot 10^{9}$ & $1.05 \cdot 10^{9}$ \\
\hline$\Sigma \boldsymbol{\ell}$ & $3.08 \cdot 10^{6}$ & $3.95 \cdot 10^{6}$ & $1.86 \cdot 10^{6}$ & $1.79 \cdot 10^{6}$ & $1.88 \cdot 10^{6}$ \\
\hline
\end{tabular}

The overall control performance, i.e., in terms of cost minimization, is assessed based on ten identical experiments, after which the data are postprocessed. The detailed results are combined in Table IV (300 rpm) and Table V (1500 rpm). In the former scenario, $\mathrm{ADP}^{1}$ has the superior performance, outperforming all other model-based algorithms despite its reduced computational burden. A $89.7 \%$ better control is achieved when compared to MPC ${ }^{1}$, mainly due to the more accurate actuation in terms of both torque and flux. For higher speeds, its control performance is competitive with $\mathrm{MPC}^{2}$ and $\mathrm{MPC}^{3}$. MPC ${ }^{1}$ is outperformed by $52.4 \%$. The diminished progress can be caused by slight parameter variations or more expressed iron losses at higher speeds as these losses are not taken into account in the 

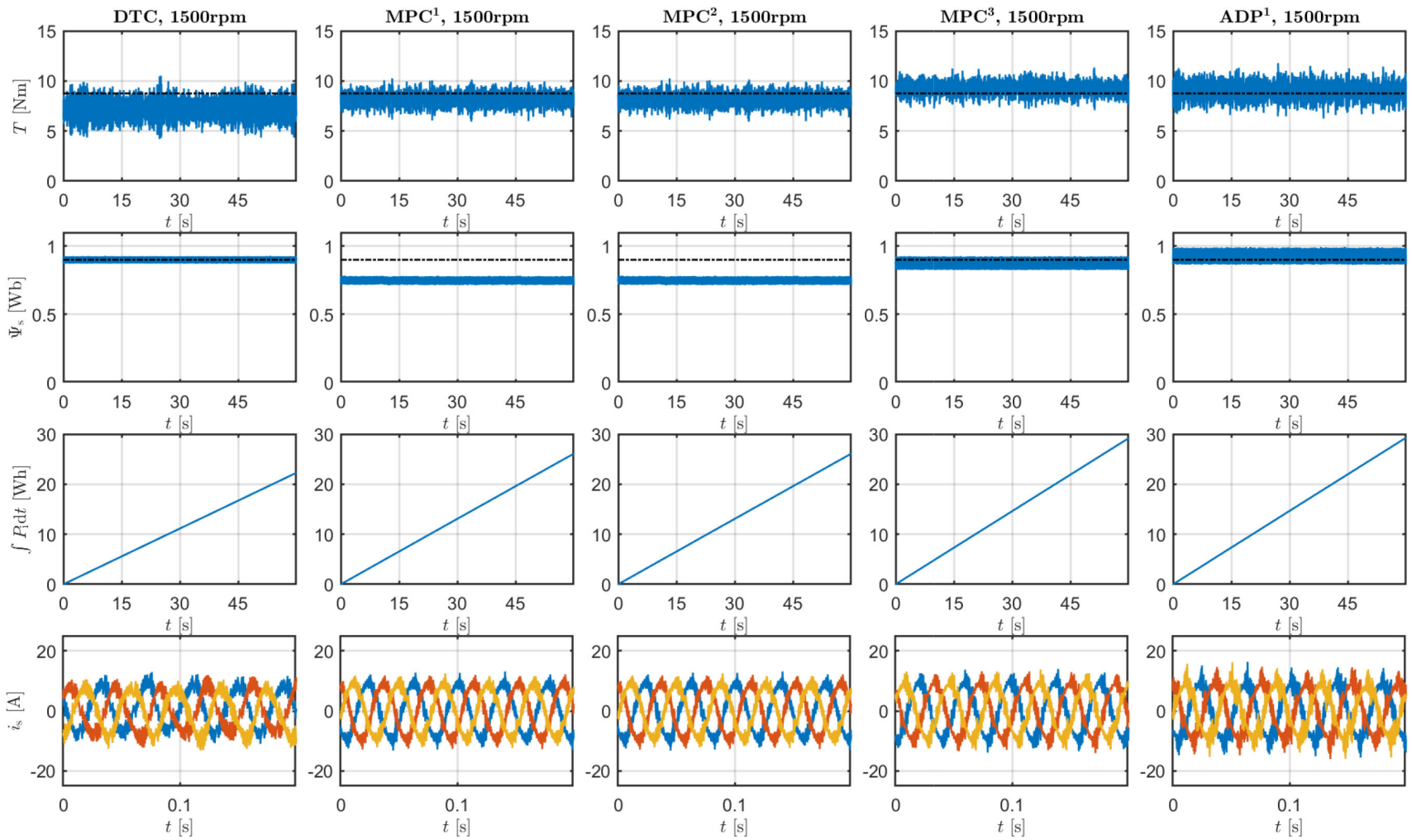

Fig. 4. Experimental steady-state response of representative quantities, i.e., torque, stator flux, consumed energy, and stator current components, for the considered $5.5 \mathrm{~kW}$-motor rotating at $1500 \mathrm{rpm}$ and actuated using the five alternative controllers.
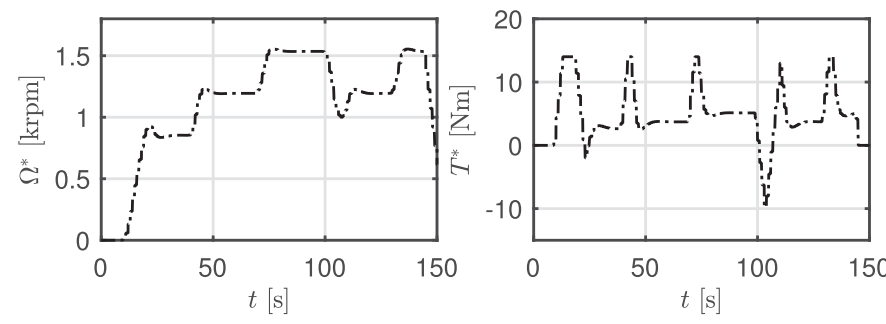

Fig. 5. Dynamic loading scenario for the experimental validation. The speed profile is imposed by the external load motor.

models. The difference between MPC ${ }^{2}$ and MPC $^{3}$ is also less pronounced, hinting at less accurate analytical models.

2) Dynamic Loading Scenario: A highly variable load in both the speed and the torque demand, visualized in Fig. 5, is now imposed. This load cycle can be considered as a representative reflection of a possible industrial application.

The time responses of the torque, stator flux magnitude, and total energy consumption are provided in Fig. 6 for the distinct controllers. These quantities have a direct impact on the total cost of control. The associated references are visualized by a dashed line. For standard MPC with a small prediction horizon $N$, one can once more observe a persistent underactuation in torque and flux in order to reduce the energy consumption. The associated negative impact on the long-term is not taken into account as a result of the limited prediction horizon. The proposed $\mathrm{ADP}^{1}$ consequently yields the most accurate actuation by using the value function approximation as a reliable reflection of
TABLE VI

Measured Averaged Energy EfFiciency of the $5.5 \mathrm{~kW}$-Motor SUBJECT TO A DYNAMIC LOADING SCENARIO

\begin{tabular}{c||c|c|c|c|c}
\hline & DTC & MPC $^{\mathbf{1}}$ & MPC $^{\mathbf{2}}$ & MPC $^{3}$ & $\mathbf{A D P}^{\mathbf{1}}$ \\
\hline \hline $\boldsymbol{\eta}$ & $70.2 \%$ & $71.8 \%$ & $75.3 \%$ & $77.2 \%$ & $73.3 \%$ \\
\hline
\end{tabular}

long-term behavior. When the power demand is zero during the initial stage of the cycle, the controller nevertheless delays the flux build-up to save energy.

The overall controller performance in terms of cost minimization is then assessed. Based on ten experiments, the average partial costs are processed and visualized in Fig. 7 together with the total summed cost over the trajectory. One can once more observe that the total cost for the $\mathrm{ADP}^{1}$ is the lowest, hence proving the usefulness of the control strategy in practical industrial applications. The computationally demanding $\mathrm{MPC}^{3}$ is beaten by $13.8 \%$, mainly due to a more accurate flux tracking. $\mathrm{MPC}^{1}$, with a similar latency as $\mathrm{ADP}^{1}$, falls back by $86.2 \%$. The associated torque and flux trajectories differ significantly from the desired references, as already highlighted based on Fig. 6 .

Despite the significantly improved overall performance, the total energy consumption $\Sigma \ell_{\mathrm{P}}$ tends to grow. This can however be compensated by a more accurate actuation and hence an increase in mechanical power. The measured energy efficiency $\eta$ is therefore provided in Table VI. The energy efficiency is defined as the ratio of the integrated mechanical power and the total consumed energy. The efficiency is slightly increased when incorporating the value function approximation. Multistep approaches nevertheless provide more appealing prospects in 

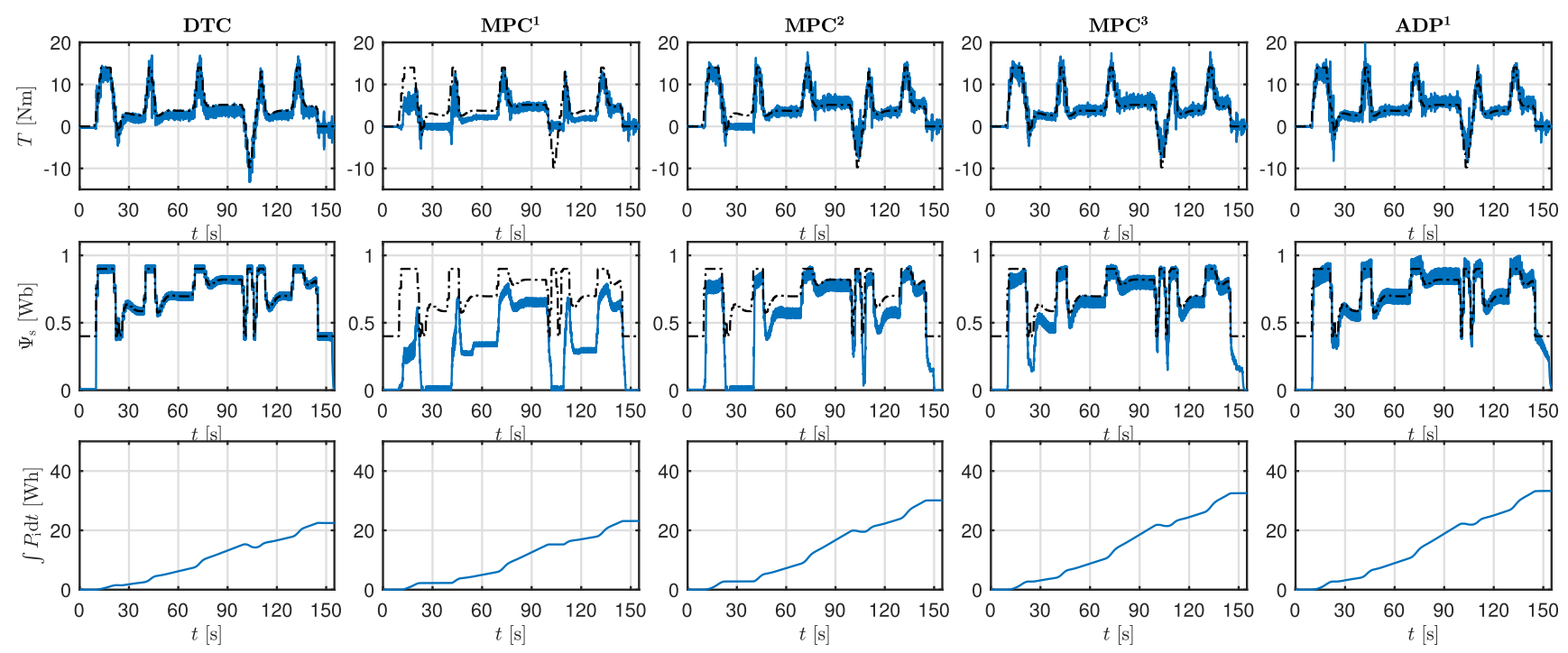

Fig. 6. Experimental response of representative quantities, i.e., torque, stator flux, and consumed energy, for the considered $5.5 \mathrm{~kW}$-motor subject to a dynamic loading scenario and actuated using the five alternative controllers.

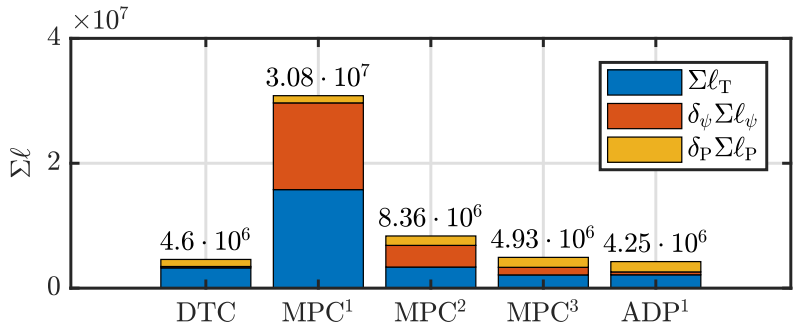

Fig. 7. Averaged experimental cost contributions and total cost for the $5.5 \mathrm{~kW}$-motor subject to a dynamic loading scenario.

terms of purely energetic performance. Multistep ADP can therefore be the topic of future research in the domain of energyefficient actuation. $\mathrm{ADP}^{1}$ nevertheless already yields the most promising overall cost of control as denoted in Fig. 7, which is the primary performance criterion when benchmarking control algorithms.

As a final remark, it is important to stress that, although not modeled, losses in the semiconductor power switches are inherently present in the gathered experimental data as the total input power drawn from the dc-source is measured.

\section{CONCLUSION}

Recent trends in electric drive technology indicated the growing importance of energy-efficient operation. Energetic considerations should nevertheless always be combined with accurate actuation in terms of torque and flux tracking. A multiobjective control problem was hence attained, which can be optimized using finite-set MPC. Due to the computational burden associated to long-term predictions, a real-time execution, i.e., at the frequency of the semiconductor power switches in the drive, was complicated. This article therefore proposed a real-time optimization framework for IM drives relying on ADP. The expected impact of instantaneous control decisions was summarized by a mathematically favorable value function approximation. This value function approximation was determined in an offline stage, which required the cost function to be quadratic in the states. The definition of an augmented state vector was hereby necessary. As only discrete switching combinations were considered, the online optimization problem was reduced to a brute force enumeration of a limited amount of possible scenarios. Single-step predictions were thus combined with long-term information in the shape of the value function approximation. A physical realization on a prototyping FPGA showed that the associated latency is comparable to state-of-the-art single-step MPC. Experimental results in both steady-state and dynamic conditions nevertheless indicated a remarkable progress in terms of the global control performance, leading to improvements of up to $89.7 \%$ when comparing single-step ADP with regular MPC. The developed methodology, combining real-time feasibility with accurate control, may hence serve a wide range of practical IM drive applications.

\section{ACKNOWLEDGMENT}

The authors would like to thank BOF 01N02716 and the Flanders Make projects DIRAC and EMODO.

\section{REFERENCES}

[1] N. P. Quang and J.-A. Dittrich, Vector Control of Three-Phase AC Machines: System Development in the Practice (Series Power Systems), 2nd ed. Berlin, Germany: Springer, 2015.

[2] J.-F. Stumper, A. Dötlinger, and R. Kennel, "Loss minimization of induction machines in dynamic operation," IEEE Trans. Energy Convers., vol. 28, no. 3, pp. 726-735, Sep. 2013.

[3] P. Vas, Sensorless Vector and Direct Torque Control.London, U.K.: Oxford University Press, 2003.

[4] C. Lascu, S. Jafarzadeh, M. S. Fadali, and F. Blaabjerg, "Direct torque control with feedback linearization for induction motor drives," IEEE Trans. Power Electron., vol. 32, no. 3, pp. 2072-2080, Mar. 2017.

[5] I. M. Alsofyani, Y. Bak, and K.-B. Lee, "Fast torque control and minimized sector-flux droop for constant frequency torque controller based DTC of induction machines," IEEE Trans. Power Electron., vol. 34, no. 12, pp. 12 141-12 153, Dec. 2019. 
[6] A. H. Abosh, Z. Q. Zhu, and Y. Ren, "Reduction of torque and flux ripples in space vector modulation-based direct torque control of asymmetric permanent magnet synchronous machine," IEEE Trans. Power Electron. vol. 32, no. 4, pp. 2976-2986, Apr. 2017.

[7] K.-K. Shyu, J.-K. Lin, V.-T. Pham, M.-J. Yang, and T.-W. Wang, "Global minimum torque ripple design for direct torque control of induction motor drives,"' IEEE Trans. Ind. Electron., vol. 57, no. 9, pp. 3148-3156, Sep. 2010.

[8] Z. Zhang, R. Tang, B. Bai, and D. Xie, "Novel direct torque control based on space vector modulation with adaptive stator flux observer for induction motors," IEEE Trans. Magn., vol. 46, no. 8, pp. 3133-3136, Aug. 2010.

[9] C. Patel, R. P. P., A. Dey, R. Ramchand, K. Gopakumar, and M. P. Kazmierkowski, "Fast direct torque control of an open-end induction motor drive using 12-sided polygonal voltage space vectors," IEEE Trans. Power Electron., vol. 27, no. 1, pp. 400-410, Jan. 2012

[10] G. Buja and M. Kazmierkowski, "Direct torque control of PWM inverterfed AC motors-A survey," IEEE Trans. Ind. Electron., vol. 51, no. 4, pp. 744-757, Aug. 2004.

[11] S. Vazquez, J. Rodriguez, M. Rivera, L. G. Franquelo, and M. Norambuena, "Model predictive control for power converters and drives: Advances and trends," IEEE Trans. Ind. Electron., vol. 64, no. 2, pp. 935-947, Feb. 2017.

[12] T. Geyer, G. Papafotiou, and M. Morari, "Model predictive direct torque control part I: Concept, algorithm, and analysis," IEEE Trans. Ind. Elec tron., vol. 56, no. 6, pp. 1894-1905, Jun. 2009.

[13] Y. N. Tatte and M. V. Aware, "Torque ripple and harmonic current reduction in a three-level inverter-fed direct-torque-controlled five-phase induction motor," IEEE Trans. Ind. Electron., vol. 64, no. 7, pp. 5265-5275, Jul. 2017.

[14] Y. Wang, T. Ito, and R. D. Lorenz, "Loss manipulation capabilities of deadbeat direct torque and flux control induction machine drives," IEEE Trans. Ind. Appl., vol. 51, no. 6, pp. 4554-4566, Nov. 2015.

[15] S. A. Odhano, R. Bojoi, A. Boglietti, G. Griva, and S. G. Rosu, "Maximum efficiency per torque direct flux vector control of induction motor drives,' in Proc. IEEE Energy Convers. Congr. Expo., Sep. 2014, pp. 1293-1300.

[16] J. Wang, F. Wang, Z. Zhang, S. Li, and J. Rodrìguez, "Design and implementation of disturbance compensation-based enhanced robust finite control set predictive torque control for induction motor systems," IEEE Trans. Ind. Informat., vol. 13, no. 5, pp. 2645-2656, Oct. 2017.

[17] M. Habibullah, D. D.-C. Lu, D. Xiao, and M. F. Rahman, "A simplified finite-state predictive direct torque control for induction motor drive," IEEE Trans. Ind. Electron., vol. 63, no. 6, pp. 3964-3975, Jun. 2016.

[18] W. Xie, X. Wang, F. Wang, W. Xu, R. Kennel, and D. Gerling, "Dynamic loss minimization of finite control set-model predictive torque control for electric drive system," IEEE Trans. Power Electron., vol. 31, no. 1, pp. 849-860, Jan. 2016.

[19] O. Sandre-Hernandez, J. Rangel-Magdaleno, and R. Morales-Caporal, "A comparison on finite-set model predictive torque control schemes for PMSMs," IEEE Trans. Power Electron., vol. 33, no. 10, pp. 8838-8847, Oct. 2018.

[20] J. Rodriguez et al., "State of the art of finite control set model predictive control in power electronics," IEEE Trans. Ind. Informat., vol. 9, no. 2 pp. 1003-1016, May 2013.

[21] Y. Zhang, J. Zhu, Z. Zhao, W. Xu, and D. G. Dorrell, "An improved direct torque control for three-level inverter-fed induction motor sensorless drive," IEEE Trans. Power Electron., vol. 27, no. 3, pp. 1502-1513, Mar. 2012.

[22] P. Karamanakos, T. Geyer, N. Oikonomou, F. D. Kieferndorf, and S. Manias, "Direct model predictive control: A review of strategies that achieve long prediction intervals for power electronics," IEEE Ind. Electron. Mag., vol. 8, no. 1, pp. 32-43, Mar. 2014.

[23] P. Karamanakos, T. Geyer, and R. Kennel, "A computationally efficient model predictive control strategy for linear systems with integer inputs," IEEE Trans. Control Syst. Technol., vol. 24, no. 4, pp. 1463-1471, Jul. 2016.

[24] T. Geyer and D. E. Quevedo, "Performance of multistep finite contro set model predictive control for power electronics," IEEE Trans. Power Electron., vol. 30, no. 3, pp. 1633-1644, Mar. 2015.

[25] T. Geyer and D. E. Quevedo, "Multistep finite control set model predictive control for power electronics," IEEE Trans. Power Electron., vol. 29 no. 12, pp. 6836-6846, Dec. 2014.

[26] F.-Y. Wang, H. Zhang, and D. Liu, "Adaptive dynamic programming: An introduction," IEEE Comput. Intell. Mag., vol. 4, no. 2, pp. 39-47, May 2009
[27] Q. Wei, D. Liu, and H. Lin, "Value iteration adaptive dynamic programming for optimal control of discrete-time nonlinear systems," IEEE Trans. Cybern., vol. 46, no. 3, pp. 840-853, Mar. 2016.

[28] Y. Jiang and Z.-P. Jiang, "Global adaptive dynamic programming for continuous-time nonlinear systems," IEEE Trans. Autom. Control, vol. 60 no. 11, pp. 2917-2929, Nov. 2015

[29] Y. Wang, B. O’Donoghue, and S. Boyd, "Approximate dynamic programming via iterated Bellman inequalities," Int. J. Robust Nonlinear Control, vol. 25, no. 10, pp. 1472-1496, Jul. 2015.

[30] B. Stellato, T. Geyer, and P. J. Goulart, "High-speed finite control set mode predictive control for power electronics," IEEE Trans. Power Electron., vol. 32, no. 5, pp. 4007-4020, May 2017.

[31] dSpace GmbH, dSPACE MicroLabBox Product Information, 2018.

[32] Y. Zhang and H. Yang, "Two-vector-based model predictive torque control without weighting factors for induction motor drives," IEEE Trans. Power Electron., vol. 31, no. 2, pp. 1381-1390, Feb. 2016.

[33] J. Lofberg, "YALMIP : A toolbox for modeling and optimization in MAT LAB," in Proc. IEEE Int. Conf. Robot. Autom., Sep. 2004, pp. 284-289.

[34] M. ApS, The MOSEK Optimization Toolbox for MATLAB Manual. Version 8.1., 2017. [Online]. Available: http://docs.mosek.com/8.1/toolbox/ index.html

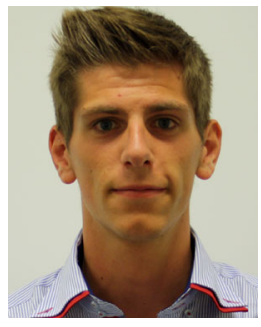

Arne De Keyser was born in Sint-Niklaas, Belgium, in 1993. He received the M.Sc. degree in electromechanical engineering, control engineering and automation and the Ph.D. degree in electromechanical engineering from Ghent University, Ghent, in 2016 and 2020, respectively.

His research interests mainly concern realtime sensing and control techniques for innovative electromechanical drivetrains. In 2016, he joined the Department of Electromechanical, Systems and Metal Engineering, Ghent University, as a Ph.D. Student of the Special Research Fund (B.O.F.).

Dr. De Keyser is also an Affiliate Member of Flanders Make, the strategic research centre for the manufacturing industry in Flanders, Belgium.

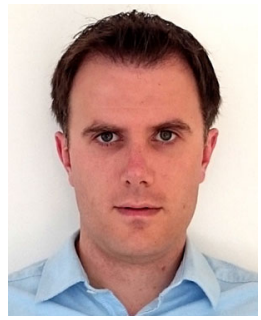

Hendrik Vansompel was born in Lokeren, Belgium, in 1986. He received the master's and $\mathrm{Ph}$.D. degrees in electromechanical engineering from Ghent University, Ghent, Belgium, in 2009 and 2013, respectively.

He is currently a Postdoctoral Research As sistant with the Electromechanical, Systems and Metal Engineering, Ghent University. His research interests include electric motors and power electronics.

Dr. Vansompel is also an affiliate member of Flanders Make, the strategic research centre for the manufacturing industry in Flanders, Belgium.

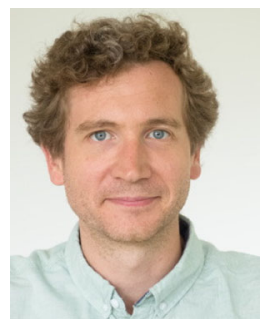

Guillaume Crevecoeur was born in Ghent, Belgium, in 1981. He received the master's and Ph.D. degrees in engineering physics from Ghent University, Ghent, Belgium, in 2004 and 2009, respectively.

He received a Research Foundation Flanders postdoctoral fellowship in 2009 and was appointed Associate Professor at the Department of Electromechanical, System and Metal Engineering from the Ghent University in 2014. He currently leads the Ghent University activities on sensing, monitoring, control and decision-making within the Flanders Make. With his team, he conducts research at the intersection of system identification, control and machine learning for mechatronic and industrial robotic systems. His goal is to endow physical dynamic systems with improved functionalities and capabilities when interacting with uncertain environments, other systems and humans.

Prof. Crevecoeur is also an Affiliate Member of Flanders Make, the strategic research centre for the manufacturing industry in Flanders, Belgium. 